\title{
¿Cómo se hace un análisis del entorno económico empresarial?
}

\author{
Carlos Parodi Trece \\ Master of Arts in Economics, Georgetown University. \\ Profesor principal del Departamento Académico de Economía e investigador \\ del Centro de Investigación de la Universidad del Pacífico, Lima, Perú
}

\section{Resumen}

La adecuada interpretación de la evolución de la economía constituye una herramienta útil para mejorar la toma de decisiones empresariales. El objetivo del artículo es explicar, con ejemplos aplicados a la realidad económica actual, cómo se hace un análisis del entorno económico y cómo este sirve para la planificación estratégica empresarial. Para ello, previa explicación conceptual del marco general, se definen el producto interno bruto, la inflación y la brecha externa como indicadores de resultados, claves en el «lenguaje» usado por los analistas. Son indicadores económicos, relacionados entre sí, que dependen de la política económica interna y de choques exógenos, definidos estos últimos como eventos que están fuera de las manos de los diseñadores de la política económica, pero que influyen en las tres variables mencionadas, como, por ejemplo, la crisis financiera internacional. A continuación sigue la formalización por medio de las identidades macroeconómicas, para finalizar explicando «cómo va la economía» mediante las mismas; así como, la relación entre el entorno económico interno con el externo. Acercar la economía a la empresa debe ser una prioridad en un contexto cada vez más integrado, caracterizado por el hecho de que lo positivo y lo negativo de lo que ocurre en la economía mundial es transmitido, mediante diversos canales, a las empresas ubicadas en distintos países. De ahí que las empresas deban ampliar su visión, pues el entorno económico no solo incluye lo que ocurre dentro del país, sino, cada vez con mayor relevancia, el devenir de la economía mundial.

Palabras clave: Entorno económico, producto interno bruto, inflación, brecha externa, crisis externas.

\section{Introducción}

Las empresas no funcionan en un vacío, sino en un entorno cambiante, inestable y poco predecible que tiene diferentes dimensiones; la económica es una de ellas ${ }^{1}$; conocer el ambiente económico en el que operan los negocios es una tarea imprescindible, pues condiciona el futuro empresa- rial. Sin embargo, ello no está exento de obstáculos: en primer lugar, comprender el significado de varios conceptos económicos que suelen ser usados por los economistas y analistas para determinar "cómo va la economía», no es una tarea simple, pues la economía como cualquier ciencia tiene un «lenguaje» propio. En segundo lugar, la rapidez de la evolución de los hechos eco-

1. Aspectos socioculturales, políticos, antropológicos, tecnológicos, etcétera, configuran otras dimensiones del entorno. 
nómicos obliga a las empresas a dedicar un importante esfuerzo en conocer su entorno.

Una vez realizada esa tarea, la empresa tiene que vincular proyecciones de las principales variables internas a la organización con el entorno en cuestión. Aunque ello no es simple, lo cierto es que en todos los planes de negocios, así como en los planes estratégicos, el punto de partida es el análisis del entorno. La pregunta es la siguiente, ¿cómo se conectan las grandes variables económicas del entorno con las proyecciones de la empresa? Por ejemplo, la crisis financiera y económica internacional iniciada en agosto de 2007 en los Estados Unidos, ha tenido repercusiones en todo el mundo; los impactos en la actividad productiva y, por ende, en los indicadores de empleo afectaron primero a los Estados Unidos, luego a Europa Occidental y, desde el primer trimestre de 2009, a América Latina. Y afectar un país significa afectar a las empresas que operan en él mismo. Como consecuencia, la misma incertidumbre altera las proyecciones empresariales. Las empresas enfrentan una serie de noticias, cuya correcta interpretación determina las decisiones tomadas.

En un mundo cada vez más interconectado («globalizado» dirían algunos), cada vez es más importante lo que ocurre más allá de las fronteras. El entorno económico local está influido, de manera creciente, por el entorno económico externo. La crisis financiera es una prueba de ello.

\section{Objetivos}

- Analizar el significado de las principales variables económicas que conforman el entorno económico dentro del cual operan las empresas.

- A partir de las variables analizadas, así como de la interrelación entre ellas, proponer una metodología de análisis del entorno económico.

\section{Marco conceptual}

\section{Globalización: ¿Amenaza u oportunidad?}

Partamos de un hecho básico: el mundo de hoy está cada día más integrado, lo que significa que las empresas operan en un entorno cada vez mayor y, por ende, más complejo. Ello constituye una oportunidad, pero al mismo tiempo una amenaza. Las empresas enfrentan el reto de cómo maximizar los beneficios de la mayor integración y cómo minimizar los costos que trae consigo. Desde fines del siglo XX, el contexto dentro del cual operan las empresas es cada vez más amplio y, por ello, «ver más allá de nuestras fronteras» es parte del análisis del entorno. Sin embargo, ¿qué es la globalización?

«Globalizar» significa llevar a escala planetaria o global alguna idea, costumbre, estilo de comportamiento, modelo de desarrollo, forma de hacer empresa, etcétera. Desde 1990 en adelante, el modelo basado en el mercado y en la apertura ha sido la norma en el mundo; es decir, se ha «globalizado» un modelo de desarrollo². Esto ha ocurrido, aun aceptando que existen distintas versiones del «capitalismo». Como consecuencia, la inversión privada ha pasado a ser el motor de la economía mundial.

Ahora bien, a pesar de que muchos escriben sobre la globalización, este término significa distintas cosas para diferentes personas. Algunos la ven como un proceso que es beneficioso, inevitable e irreversible. Otros le tienen temor, pues sostienen que aumenta la desigualdad dentro y entre las naciones, eleva el desempleo y obstaculiza el progreso social. Los cuestionamientos han aumentado en el entorno poscrisis; no obstante, quienes lo hacen, usan como sinónimos: globalización y libre mercado, igualdad discutible $^{3}$. Aun así y en términos amplios, el uso del

2. Esto no significa que exista «un» modelo de desarrollo basado en el mercado, existen matices; por ejemplo, el capitalismo coreano no es igual al estadounidense.

3. Si «globalizar» es llevar alguna idea a escala planetaria o global, nadie estaría en contra de "globalizar» la defensa de los derechos humanos. Por lo tanto, la globalización «sin apellido» es un sustantivo neutro; depende de qué se busque globalizar y cómo. 
sustantivo "globalización» en el campo económico alude a lo siguiente:

- Una mayor integración de las economías, de modo que el mundo se comporte como si fuera parte de un único mercado global. Una expresión de lo anterior es el incremento de las transacciones internacionales en los mercados de bienes y servicios, y algunos factores de producción. De ahí que sea posible dividir la globalización económica en, por lo menos, tres dimensiones: la comercial, la financiera y aqueIla relacionada con el libre tránsito de las personas entre países. La realidad muestra que algunas dimensiones están más globalizadas que otras ${ }^{4}$.

- Una ampliación del espectro dentro del cual actúan diferentes instituciones, que ahora trasciende las fronteras nacionales. La globalización económica ha ampliado el radio de acción de las empresas, los gobiernos, las instituciones internacionales y las organizaciones no gubernamentales (ONG). De ahí que como concepto, la globalización económica incluya además del crecimiento del comercio internacional, la expansión de la inversión extranjera directa (IED) ${ }^{5}$ y las corporaciones multinacionales, la integración del mercado mundial de capitales, el alcance extraterritorial de las políticas del Gobierno, una mayor atención de las ONG en problemas que se extienden por todo el mundo y las restricciones en las políticas de gobierno respecto de lo que pueden hacer, pues sus decisiones dependen cada vez más de lo que ocurra en el entorno internacional.

Por eso se ha incrementado la sensación en muchas personas que sus vidas y opciones económicas no están determinadas por ellas mismas ni por sus gobiernos, sino por fuerzas externas sobre las cuales no tienen control. En ese sentido, la globalización está redefiniendo el papel que desempeña el Estado-nación como un manejador efectivo de la economía nacional ${ }^{6}$. En adición a lo anterior, la globalización no solo es económica sino también cultural. Las personas tienden a imitar comportamientos de individuos de otros países, con el riesgo de que el modelo del «deber ser» lleve a que se pierda autenticidad, produciéndose una transferencia de actitudes, muchas veces ajena a nuestras realidades.

Sin embargo, esta ola de globalización no es la primera. En muchas formas, la economía mundial alcanzó su pico de globalización antes de la primera guerra mundial, cuando el comercio y la IED alcanzaron niveles sin precedentes, dada la tecnología y disponibilidad de transportes y comunicaciones en esa época. No obstante, la actual ola de globalización ha sobrepasado con creces a la anterior. La tasa exportaciones/PIB, que puede usarse como un indicador del incremento del comercio internacional, se multiplicó por tres entre 1950 y 1998 . En el mismo sentido, la IED creció 59\% entre 1960 y 1995. En consecuencia, está claro que en la segunda mitad del siglo $\mathrm{XX}$, tanto el comercio internacional como los flujos internacionales de capital se incrementaron de manera notable. Este proceso es aquel al que muchos autores denominan globalización (Deardoff y Stein 2000).

¿Qué ha originado estos cambios en la economía mundial? Existen básicamente dos causas: por un lado, el avance tecnológico y por el otro, los cambios en las políticas económicas. Las mejoras tecnológicas en el transporte y la comunicación han aumentado la globalización de los mer-

4. La globalización financiera, esto es la integración de los sistemas financieros nacionales con sus similares de otros países, está más avanzada que la comercial. La dimensión «menos integrada» es la laboral, dadas las barreras existentes al libre tránsito de las personas.

5. La inversión extranjera directa se define como aquel tipo de inversión productiva realizada por agentes económicos externos, en oposición a la especulativa. Mientras que la primera es más estable por el compromiso de largo plazo que lleva inmersa, la segunda es de más rápida reversión. De ahí, la deseabilidad de la primera sobre la segunda.

6. Boyer y Drache (1996). También se sugiere revisar, Schuldt (1998) y Parodi Trece (2005). 
cados, tendencia que se incrementa cada día más con el uso de Internet. Las políticas económicas también han cambiado, pues las economías cada vez son más abiertas. Ambas tendencias han sido muy claras después de la segunda guerra mundial, pero han aumentado en importancia en las últimas dos décadas.

Entonces, ¿toda la globalización significa mayor comercio y flujos de capitales originados en las mejoras tecnológicas y en la apertura de las economías? No, al mismo tiempo existen otros aspectos de la vida económica que también se han globalizado. Los gobiernos están cada vez más preocupados por las políticas que llevan a cabo otros gobiernos, pues estas afectan al mismo flujo internacional de bienes y de capitales. Las ONG cada vez ven más allá de las fronteras de sus países; algunas veces porque los problemas que enfrentan son de naturaleza global y otras, porque consideran que el papel que desempeñan tiene importancia mundial (por ejemplo, la defensa de los derechos humanos). Por medio de la IED, las corporaciones no solo operan más allá de las fronteras nacionales, sino que han crecido en tal magnitud que logran influir en los gobiernos nacionales; si uno de ellos les pone algún tipo de restricción, simplemente buscan otro país. Esta necesidad de mantener atractivo al país plantea una amenaza, pues cualquier coyuntura negativa interna puede hacer que los capitales huyan en manada. Otros autores sostienen que este hecho, por el contrario, obliga a los países a actuar en forma disciplinada, con reglas de juego estables, de modo de no salir del concierto mundial.

Otra amenaza que plantea el nuevo entorno mundial, caracterizado por la mayor integración de las economías, son los efectos que tiene una crisis en un país sobre los demás. En la medida que las empresas de los distintos países están más interconectadas, resulta cada vez más difícil evitar el "contagio»; de ahí que tener en cuenta las crisis de otros países debe estar en la agenda del análisis del entorno de cualquier empresa.
Principales variables del entorno económico

Una adecuada comprensión del entorno económico requiere el manejo de varios conceptos usados por los especialistas para analizar «cómo va la economía». Con la finalidad de proporcionar al lector un marco general que posibilite comprender el «lenguaje» utilizado, conviene mencionar que, en un nivel general, la evolución de cualquier economía puede analizarse a partir del seguimiento de tres indicadores macroeconómicos: la evolución del producto interno bruto (PIB), la inflación y la brecha externa. En los tres casos existe un comportamiento deseable de los mismos, que puede diferir de lo que efectivamente ocurra con ellos.

Las tendencias de los tres indicadores mencionados son el resultado de dos fuerzas que interactúan entre sí: la política económica interna de cada país y los choques exógenos. Estos últimos se definen como eventos que están fuera del control de los diseñadores de la política económica; por ejemplo, el Fenómeno «El Niño», la variación de los precios internacionales de las materias primas y el impacto de las crisis externas. Cuanto más cerrada sea una economía, mayor relevancia tendrá la política económica interna; sin embargo, en la realidad actual aquello solo es una ficción. La mayor interdependencia de las economías, en un mundo cada vez más globalizado, ha llevado a que los choques exógenos adquieran particular relevancia, sin que ello signifique dejar de lado un manejo adecuado de las políticas económicas internas. No obstante, parece que cada vez es menos lo que se puede hacer desde dentro, pues los impactos de lo que ocurre en el exterior desestabilizan a las economías en tal magnitud, que obliga a implementar medidas que de otro modo no se hubieran adoptado. En estos tiempos hay que preocuparse más de los fenómenos que ocurren fuera de las economías.

Sin embargo, en la medida que todo sistema económico tiene como fin último elevar el bienestar 
de los habitantes de un país, lo que conecta a los tres indicadores con el bienestar es la tendencia del empleo y de los programas sociales. Por lo tanto, la necesidad de mantener un crecimiento económico alto y estable, una inflación baja y unas cuentas externas en equilibrio (o, por lo menos, cercanas a él) no son un fin en sí mismo, sino un medio para lograr aumentos sostenidos en los niveles de bienestar.

La comprensión de este marco general permite tener una visión más global de lo que ocurre dentro de una economía en algún momento del tiempo, sin dejar de lado que la economía no evoluciona en el vacío, sino que también impactan aspectos institucionales, políticos, históricos, geográficos, culturales e internacionales. Ciertamente, dentro del entorno económico existe un número mayor de indicadores; no obstante, con los mencionados, que son indicadores de resultados económicos, es posible responder a la pregunta «¿cómo está la economía?». Una aclaración resulta pertinente: las tres variables mencionadas son «de resultado» y no de política económica; dicho de otro modo, las variables de política, como, por ejemplo, el gasto público, los impuestos, el tipo de cambio ( $y$, en general, el régimen cambiario) y los aranceles explican las tres variables de resultado. Las variables de política serán analizadas de manera somera para no desviar al lector del objetivo central.

El producto interno bruto (PIB): aspectos conceptuales

EI PIB es un indicador de la producción de una economía; mide el valor de todos los bienes y servicios finales producidos dentro de los límites geográficos de una economía, en un período determinado. Es distinto del concepto del producto nacional bruto (PNB), que mide lo producido solo por agentes económicos de un país dentro o fuera del mismo. En el caso peruano, el PIB desde 1950 ha sido mayor que el PNB, lo que refleja que las empresas e individuos extranjeros en el país generaron ingresos superiores a los que sus similares peruanos produjeron en el exterior.

Por lo general, el aumento del PIB real de un período a otro se denomina crecimiento económico y su disminución, recibe el nombre de recesión y se expresa en variaciones porcentuales. Por ejemplo, cuando se afirma que el PIB de la economía peruana creció $9,8 \%$ en 2008 , significa que la producción del país aumentó en 9,8\% comparando 2008 con 2007.

Sin embargo, el PIB real es simplemente un indicador de cambios en la producción y no necesariamente mide las variaciones en el bienestar de los individuos de una sociedad. Tampoco lo hace el PIB por habitante, pues no muestra cómo está distribuido el ingreso entre los individuos, sino que asume que todos producen lo mismo; es decir, supone una distribución de ingresos homogénea. Desde esa óptica, los incrementos en el PIB son un medio para lograr la elevación del bienestar de una sociedad, que se mide por medio de otros indicadores: por ejemplo, los niveles de pobreza, la tasa de mortalidad infantil, la tasa de analfabetismo, el nivel educativo, etcétera.

Inclusive, el Programa de las Naciones Unidas para el Desarrollo (PNUD) calcula, desde 1990, el índice de desarrollo humano $\left(\right.$ IDH ${ }^{7}$. Este es un indicador compuesto que mide el avance promedio de un país, construido a partir de tres dimensiones básicas del desarrollo humano: el PIB por habitante, la esperanza de vida al nacer y la tasa de alfabetización de los adultos, y la tasa bruta combinada de matrícula en la educación primaria, secundaria y terciaria. La idea es agre-

7. En el Informe de Desarrollo Humano del año 2008, de un total de 177 países, Perú figura en la ubicación 87 y es considerado un país de desarrollo humano medio. Entre los países de América Latina, el país mejor ubicado es Argentina (puesto 38). En el ámbito mundial, Islandia y Noruega son los países con mayor índice de desarrollo humano. Para construir el índice, el PNUD usó datos del año 2005. Cabe mencionar que dada la crisis financiera internacional y su impacto sobre Islandia, es probable que en informes que insuman información más reciente, dicho país se ubique varios escalones atrás. Véase, <http://www.undp.org>. 
garle dimensiones humanas a las dimensiones materiales que se miden por medio del PIB. Si bien no mide el desarrollo en una acepción amplia, sin duda es un avance. Desde 1997, el PNUD también calcula el índice de pobreza humana, para lo cual utiliza los siguientes indicadores: porcentaje de personas que se estima morirán antes de los cuarenta años, porcentaje de adultos que son analfabetos, porcentaje de personas sin acceso a servicios de salud y agua potable, y porcentaje de niños menores de cinco años con peso insuficiente.

El comportamiento del PIB real en el tiempo, por lo general, no es suave, sino fluctuante, en torno de una tendencia. Esas fluctuaciones de corto plazo, que en oportunidades pueden ser bastante pronunciadas, se denominan ciclos económicos. Los ciclos económicos tienen dos fases: una de expansión, por encima de la tendencia, seguida de una recesión, por debajo de la misma. Uno de los objetivos de una política de estabilización, que forma parte de la política económica, es justamente aplanar el ciclo, de modo de hacer más estable y predecible la evolución del PIB.

EI PIB real suele analizarse desde dos ópticas: por el lado de la demanda, es decir, ¿quién compra el PIB?; y por el lado de la oferta, que analiza a los sectores de la economía que contribuyen con el PIB. Estas dos visiones son claves para determinar qué sectores son los que crecen más y a quién le venden esos sectores. La información mencionada es crucial para el análisis del entorno económico.

Por el lado de la demanda, esta se divide en demanda interna y demanda externa (exportaciones); a su vez, la demanda interna, se subdivide en consumo privado, consumo público e inversión bruta interna, dividida en privada y pública. La suma de la demanda interna más la demanda externa es igual a la oferta global, que a su vez es el resultado de sumar el PIB (oferta interna) más las importaciones.

Por el lado de la oferta, el PIB por sectores económicos se divide en agropecuario (agrícola más pecuario), pesca, minería e hidrocarburos, manufactura, electricidad y agua, construcción, comercio y otros servicios. La variación en el PIB real global es un promedio ponderado de los cambios en el PIB de cada sector económico.

¿Cuál debe ser el objetivo de la política económica con respecto del PIB real? Un crecimiento ascendente, lo menos cíclico posible y sano. Esto último significa que no genere presiones inflacionarias ni desequilibrios en otras cuentas ${ }^{8}$.

¿Qué es una recesión? Una complicación al análisis del entorno económico

Ante la crisis financiera internacional, la mayoría de gobiernos del mundo ha respondido de dos maneras: con una política monetaria expansiva (una menor tasa de interés para abaratar el crédito) y con una política fiscal expansiva (programas de aumentos en el gasto público). Sin pretender entrar en los detalles, que escapan a los fines de la presente investigación, ello ha sido presentado como respuesta a la recesión.

Sin embargo, ¿existe consenso respecto de la definición de una recesión? La definición tradicional de «recesión» destaca por su simpleza: dos trimestres consecutivos de reducción en el PIB, pero comparado contra el trimestre inmediato anterior. Dicho de otro modo, en las economías avanzadas, la evolución del PIB no se calcula contra «igual período del año previo», como ocurre en el Perú, sino contra el período inmediato anterior. Existen otras diferencias como, por ejemplo, que en el Perú existen cifras mensuales del PIB, mientras que en los Estados Unidos, solo trimestrales.

8. Por ejemplo, un incremento exagerado del PIB puede llevar a un aumento en las importaciones, por las necesidades de la industria. Si las exportaciones no se elevan al mismo ritmo, se genera una brecha externa. Una brecha externa alta, como se verá luego, no es sostenible en el largo plazo. 
Entonces, ¿está o no el mundo en recesión? En los Estados Unidos, la entidad oficial de anunciar la recesión es el Consejo Nacional de Investigación Económica (National Bureau of Economic Research-NBER), que tiene otra definición de recesión: «a significant decline in activity spread across the economy, lasting more than a few months, visible in industrial production, employment, real income and whole-retail trade» ${ }^{9}$. Por lo tanto, usan cuatro indicadores: empleo, producción industrial, ingreso personal real y volumen de ventas de los sectores manufactureros y comerciales al por menor. No indican los umbrales, lo que le confiere algún nivel de subjetividad; por ejemplo, ¿a cuánto debería aumentar el desempleo para «declarar» recesión? ¿Y los demás indicadores? La entidad en cuestión declaró que la recesión en los Estados Unidos comenzó en diciembre de 2007, cuando los indicadores de evolución trimestral del PIB aún eran positivos. De acuerdo con la visión convencional, dicho país habría entrado en recesión en el cuarto trimestre de 2008. La cifra de $-6,0 \%$ (comparada contra el tercer trimestre) se repitió en el primer trimestre de 2009 (en comparación con el último trimestre de 2008).

La ausencia de consenso es relevante para el análisis del entorno económico. Si un gobierno anuncia una recesión, entonces las empresas esperarán que sean implementadas diversas medidas para combatirla y ello altera el entorno económico futuro. Sin embargo, el anuncio lleva implícita una definición de recesión que no es común para todos los países.

\section{La inflación}

La inflación es la tasa de aumento en el nivel de precios. Dos aclaraciones son pertinentes: en primer lugar, no es igual a precios altos, sino a la variación en ellos; en segundo lugar, bajar la inflación no significa que los precios bajen, sino que suban menos. La autoridad monetaria responsable del control de la inflación es el Banco Central.

En el caso peruano, de acuerdo con la Constitución Política (capítulo V, artículo 84), el Banco Central tiene como objetivo único «preservar la estabilidad monetaria», para lo cual goza de autonomía dentro de los límites establecidos por su propia Ley Orgánica. El logro de la estabilidad monetaria significa que el Banco Central cumpla con la meta anual anunciada: $2 \%$ anual con un margen de error de $1 \%$ por encima o por debajo de la misma ${ }^{10}$. En esa dirección, diseña la política monetaria, que consiste en inducir el comportamiento de la tasa de interés de referencia que, en términos simples es la tasa de interés interbancaria. Es decir, la tasa de interés que los bancos se cobran entre sí por préstamos de muy corto plazo.

Más allá de los detalles, desde el punto de vista del análisis del entorno económico, la pregunta es la siguiente: ¿por qué es importante la estabilidad monetaria? Por las siguientes razones:

- Cuando las empresas buscan un nicho de mercado para ampliar sus actividades, ¿qué observan? Lugares donde exista escasez del bien o servicio en cuestión. La escasez se expresa en ligeros aumentos del precio. En otras palabras, el mayor precio, reflejo de la escasez, es la señal recibida por la empresa. Sin embargo, en caso exista inflación «la señal se nubla» y no es posible saber si el aumento en el precio es debido a la inflación (en cuyo caso no habría que emprender la actividad) o a la escasez (caso en el cual sí habría que iniciar el negocio). La decisión usual ante la disyuntiva e incertidumbre es no invertir.

9. La definición puede encontrarse en <http://www.nber.org>.

10. Para la consecución de la meta, el Banco Central sigue el esquema de metas explícitas de inflación (del inglés, inflation targeting) desde el año 2002. Hasta 2006, la meta era de 2,5\% anual +/- 1\%; desde 2007 fue ajustada a $2 \%+/-1 \%$. 
- La inflación distrae recursos que son asignados a otras actividades, como la especulación.

- Disminuye el poder adquisitivo, afectando en mayor medida a los segmentos de bajos ingresos.

- Atenta contra la marcha económica del país, pues no permite que la moneda cumpla con sus funciones: depósito de valor, unidad de cuenta y medio de cambio. En el proceso y de manera gradual, la moneda nacional es reemplazada por otro activo. En el caso peruano, dada la trayectoria inflacionaria de los años 1980, el reemplazo fue el dólar.

La evolución de la inflación en el Perú ha sido consistente con la meta. En 2002 fue de 1,5\%, en $2003 ; 2,5 \%$, en $2004 ; 3,5 \%$, en $2005 ; 1,5 \%$, en $2006 ; 1,1 \%$, en $2007 ; 3,9 \%$ y en 2008 , $6,7 \%{ }^{11}$. Entre 2002 y 2006, la inflación anual estuvo dentro de los márgenes establecidos por el Banco Central. En 2007 y 2008, estuvo por encima de la meta, lo cual tuvo su origen en los choques externos negativos: el aumento en el precio del petróleo y las elevaciones de los precios de los alimentos, en especial en el primer semestre de 2008. Nuevamente, el entorno externo afectó al interno. En 2009, la inflación retornó a la meta al reducirse las presiones inflacionarias provenientes del exterior.

\section{La brecha externa y las crisis externas}

La brecha externa se «lee» en la balanza de pagos, que es un registro que resume todas las transacciones comerciales y financieras realizadas por residentes de un país y el exterior. Para fines de la presente investigación, presentaremos una versión simplificada de la balanza de pagos. Está dividida en dos partes: la balanza en cuenta corriente y la balanza de capitales. En la primera se registran las exportaciones e importaciones de bienes y servicios; mientras que en la segunda, los movimientos de capitales, por ejemplo: la inversión extranjera directa y la deuda externa, pública y privada.
La brecha externa equivale a un déficit en la balanza en cuenta corriente y, usualmente, es medida como porcentaje del PIB. En términos simples, la brecha externa significa que un país, durante un período, ha importado más de lo que ha exportado. Surge la pregunta siguiente: si un país importa más de lo que exporta (sale más dinero del que ingresa), ¿de dónde financia la diferencia? La respuesta se encuentra en la balanza de capitales. El ingreso de capitales financia la brecha externa. Un ejemplo aclara lo anterior. Una empresa extranjera decide invertir en un país, con lo cual se convierte en residente en el país en cuestión. La inversión extranjera queda registrada en la balanza de capitales como un ingreso de capitales. Si la mencionada empresa decide importar algún insumo para producir, entonces, en la balanza de pagos aumentan las importaciones, ampliándose la brecha externa o déficit en la cuenta corriente. Dicho de otro modo, el ingreso de capitales, registrado en la balanza de capitales, «sirvió» para importar el insumo requerido, con lo cual ha financiado el exceso de importaciones sobre exportaciones; es decir, la brecha externa. De no haber importado el insumo, no hubiera sido posible producir.

Los problemas pueden aparecer cuando una economía depende en exceso del ingreso de capitales; ¿la razón?, una eventual reversión, es decir, salida de capitales del país. La crisis externa alude a la salida abrupta y repentina de los capitales extranjeros de una economía, lo cual implica que no hay cómo financiar las importaciones y se reduzca el crecimiento económico.

Veamos esto con mayor detalle. El crecimiento económico de un país depende de la inversión. De ahí que sea un hecho estilizado que los países con mayores niveles de inversión hayan logrado crecer más rápido, siempre y cuando los altos niveles de inversión hayan sido bien utilizados, además de ser perdurables en el tiempo. La condición previa para lograr altos niveles de inversión es que existan los recursos su-

11. Banco Central de Reserva del Perú, consulta en línea, <http://www.bcrp.gob.pe>. 
ficientes para hacerlo, y ellos provienen del ahorro. El sistema financiero canaliza el ahorro hacia la inversión, de modo que se genera un círculo virtuoso entre mayor ahorro, mayor inversión y mayor crecimiento.

Sin embargo, los países en desarrollo se han caracterizado por tener un bajo nivel de ahorro interno, medido como porcentaje del PIB. Entonces, son países que sufren de una escasez crónica de ahorro ${ }^{12}$, razón por la cual han tenido que recurrir al ahorro externo para poder complementar sus bajos niveles de ahorro interno. El ahorro externo es captado por estos países mediante diversos mecanismos; uno de ellos es la obtención de préstamos del exterior, dirigidos al sector público (como ocurrió en América Latina hasta la década de los años 1980) o al sector privado (década de los años 1990 en adelante). Resulta crucial tener claro que si bien esta dependencia del ahorro externo no es negativa en sí (en la medida que el uso de los recursos externos permita luego su respectivo repago), genera compromisos futuros de pago con el exterior. Otro mecanismo, como se ha visto, es la inversión extranjera, que admite diversas variantes; en cualquier caso, se trata de un ingreso de capitales.

Desde inicios de la década de los años 1990, la apertura de las economías ocurrió en dos niveles: por un lado, una tendencia a reducir las barreras al comercio internacional, de modo de incrementar tanto las exportaciones como las importaciones de bienes y servicios. Por otro lado, la apertura financiera significó que las economías emergentes pudieran acceder a ingentes cantidades de recursos externos (entrada de capitales privados), que permitieron ciclos expansivos de crecimiento de estas economías. Apareció así un ciclo virtuoso: el ingreso de capitales (ahorro externo) aumentó la inversión y la demanda interna y las economías comenzaron a crecer. Sin duda, para que este ingreso de capitales pudiera materializarse hubo factores internos (como la estabilización y las reformas estructurales), así como externos (como la caída de la tasa de interés de la Reserva Federal de los Estados Unidos, que empujó a los capitales hacia plazas más rentables y la recesión en las economías industrializadas).

Sin embargo, algo ocurrió. Así como el ingreso de capitales aceleró el crecimiento, también ocasionó una dependencia de los mismos, de modo que cualquier reversión de ellos no solo frenaría el crecimiento, sino también obligaría a los países a repagar sus deudas en un contexto de menor crecimiento. Las crisis financieras de los años 1990 se asocian con esa reversión abrupta de la dirección de los capitales: de un contexto caracterizado por su abundancia se pasó a una sensible disminución. Los casos de México en 1994, Asia Oriental en 1997 y de Rusia en 1998 son los más saltantes, tanto por la importancia de esas economías como porque se produjo el denominado efecto contagio al resto de economías emergentes. Los capitales no solo huyeron de esos países sino, en general, de todas las economías emergentes, a pesar de que la mayoría de ellas mantenía las finanzas públicas en equilibrio y bajos niveles de inflación ${ }^{13}$. En síntesis, la reversión de los capitales provocó una crisis de pagos que llevó a paquetes de rescate internacional de grandes dimensiones, cuyo primer objetivo era que los países contaran con los recursos para poder cumplir con sus obligaciones externas. El objetivo de fondo era que los inversionistas externos retomaran la confianza en los países afectados, de manera que el ingreso de capitales retorne a aquellos países. De ahí que los paquetes de rescate estuvieran condicionados a la implementación de una serie de reformas estructurales.

Las crisis externas, vistas desde esta óptica, tuvieron varios aspectos. En primer lugar, precipi-

12. En Asia Oriental, varias economías no tenían esta característica, pero aún así hicieron crisis en 1997. Esto implica que los altos niveles de ahorro e inversión no garantizan que un país evite una crisis.

13. El equilibrio en las finanzas públicas significa que los ingresos del gobierno son mayores o iguales que sus gastos. El déficit fiscal aparece cuando los gastos son mayores que los ingresos. No debe confundirse con la brecha externa, que, como se ha visto, aparece cuando el país importa más de lo que exporta. 
taron crisis cambiarias, pues al salir los capitales, se produjo una escasez de dólares que presionó al tipo de cambio nominal, es decir, «el dólar subió de precio» ${ }^{14}$. Las autoridades de los países optaron por una defensa del tipo de cambio, mediante la venta de dólares, para compensar la salida de capitales. En ese sentido, usaron sus reservas. Sin embargo, cuando estas se agotaron, no tuvieron otra alternativa que abandonar la defensa del tipo de cambio y dejar su determinación al libre mercado, lo que supuso fuertes aumentos del mismo.

En segundo lugar, las crisis externas también se convirtieron en crisis bancarias y financieras. La interrupción del ingreso de capitales (en especial con la crisis rusa de 1998 ) provocó que los bancos privados no pudieran hacer frente a sus pagos con el exterior, porque la mayoría de las deudas habían sido contraídas a corto plazo; además, se endeudaban en dólares, pero prestaban internamente en moneda nacional. La crisis cambiaria (que llevó a que los agentes económicos internos requirieran de más unidades de moneda nacional para comprar dólares y pagar a los bancos), unida a la contracción de liquidez en moneda nacional (al vender dólares para contrarrestar la salida de capitales, las autoridades monetarias retiraban liquidez del sistema), hizo muy difícil que los bancos contaran con la disponibilidad de moneda extranjera para cumplir con sus pagos. Por ello, gran parte de los recursos obtenidos de los paquetes internacionales de rescate se dirigieron a facilitar el repago a los bancos privados, por el temor de una desestabilización del sistema financiero mundial.

Si bien es cierto cada país tiene particularidades diferentes, la descripción anterior corresponde a hechos estilizados, comunes a todos ellos.
Los capitales que huyeron de estos países buscaron plazas más seguras y las encontraron en los Estados Unidos y Europa Occidental. El enorme ingreso de capitales financió, a inicios del siglo XXI, el exceso de gasto y sobreendeudamiento de las familias y las instituciones financieras. Pocos años después estallaría la crisis de los Estados Unidos en 2007. Más allá de los detalles, la lección es clara: el entorno económico de un país o grupo de países no puede analizarse de manera independiente; para bien o para mal, el mundo está más interconectado y en la "solución» a una crisis pueden encontrarse las semillas de la crisis siguiente.

\section{Metodología}

Mediante el uso de información del mundo real, se aplicarán los conceptos revisados, así como la interrelación entre ellos. Se presentan dos aplicaciones: la primera supone, como paso previo, una formulación cuantitativa de la relación entre las variables. Luego, usando el marco presentado, se explica cómo puede surgir una crisis externa y qué razones puede tener. La segunda aplicación utiliza información recogida de informes económicos preparados por el Banco Central de Reserva del Perú y el Fondo Monetario Internacional; explica, de manera secuencial, una forma de cómo la empresa puede realizar el análisis del entorno económico.

\section{Aplicaciones empíricas}

Estudio de las relaciones económicas a partir de las identidades macroeconómicas: las crisis externas $^{15}$

El análisis del entorno económico puede quedar formalizado mediante las denominadas identidades macroeconómicas.

14. La política cambiaria es responsabilidad del Banco Central, quien ha implementado en el Perú un sistema cambiario de flotación administrada. Esto significa que el tipo de cambio está determinado por el libre mercado, pero la autoridad monetaria interviene en el mercado cambiario para evitar «fluctuaciones bruscas»; es decir, no tiene una meta cambiaria, como en el caso de la inflación. Dicho de otro modo, cuando considera que la caída del tipo de cambio nominal ha sido brusca, compra dólares con el objetivo de aumentar su demanda y subir ¿o mantener su precio?; en el caso contrario, vende dólares. En el primer escenario aumentan las reservas y la cantidad de soles en circulación; mientras que en el segundo, disminuyen ambos.

15. Esta parte de la investigación está basada en Parodi Trece (2009: 34-45). 
En primer lugar, el análisis del PIB por el lado de la demanda (quién compra lo producido) se formaliza de la siguiente manera:

$P I B=C+I+G+X-M$

El consumo, representado por $\mathrm{C}$, indica el gasto privado de los residentes en un país. La inversión (I) significa el aumento del stock físico de capital; es decir, la cantidad acumulada por las empresas para construir nuevas fábricas, así como la adquisición de equipo y tecnología para producir más en el futuro. El gasto público (representado por $\mathrm{G}$ ) incluye las compras de bienes y servicios realizadas por el sector público. Se subdivide en gasto corriente y gasto de capital (donde se ubica la inversión pública; por ejemplo, el mantenimiento de las carreteras). Las exportaciones $(\mathrm{X})$ representan la demanda del exterior por bienes y servicios producidos dentro del país; mientras que las importaciones (M) son las compras realizadas por residentes de bienes y servicios del exterior. De ahí que en la ecuación (1) aparezcan con signo negativo. El término (X-M) es conocido como exportaciones netas $y$, en términos de las identidades macroeconómicas, como la cuenta corriente de la balanza de pagos ${ }^{16}$.

La utilidad de la descomposición anterior radica en el hecho de que permite comprender las causas de una expansión o recesión de la economía. A partir de este análisis, es posible proponer una política económica que conduzca a los resultados deseados.

Reordenando la identidad (1), se obtiene:

$P I B+M=C+I+G+X$

La expresión de la izquierda es la oferta global de bienes y servicios (lo producido internamente más lo importado), mientras que el lado derecho muestra la demanda global (o demanda agregada), que se descompone en la demanda interna $(C+I+$
G) -conocida como «Absorción» (A)- y la demanda externa -exportaciones-, que son las compras realizadas desde el exterior por producción realizada dentro del país (PIB). Un aumento en el ingreso de capitales a un país eleva la absorción, sea por la vía de mayor consumo, inversión o gasto de gobierno.

EI PIB obtenido por un país en un período es igual al ingreso nacional $(\mathrm{Y})$, definido este último como la suma de los ingresos obtenidos por los factores de producción durante dicho período. La razón de esta igualdad es simple. El uso de una unidad monetaria para comprar algún bien o servicio representa no solo un gasto para quien la realiza, sino también un ingreso para quien lo recibe. Por ejemplo, si se adquiere un paquete de galletas en tres soles, ese monto representa el valor del bien medido a precios de mercado, por lo que el PIB aumenta en ese valor. Al mismo tiempo, quien vendió el bien incrementa sus ingresos en tres soles; de esta manera, el ingreso nacional también aumenta en tres soles. Así, la igualdad entre PIB e ingreso nacional es una identidad, al igual que la ecuación (1). En lo que sigue, cuando se aluda al PIB, también se estará haciendo referencia al ingreso nacional, pues son equivalentes.

El ingreso recibido puede tener dos destinos: el consumo (C) o el ahorro (S), que es el ingreso no consumido. Para comprender mejor lo anterior, supongamos por un momento que la economía es cerrada (no comercia con el exterior) y que no existe sector público. Entonces:

$P I B=Y=C+I$

Como $\mathrm{S}=\mathrm{Y}-\mathrm{C}$, entonces, $\mathrm{S}=\mathrm{I}$. En una economía cerrada, el ahorro es idéntico a la inversión.

La extensión de este razonamiento a una economía abierta, incorporando además al sector público, lleva a que no necesariamente se cumpla que el ahorro sea igual a la inversión. La presen-

16. Como ha sido analizado y en términos simples, la balanza en cuenta corriente es la suma de la balanza comercial y la balanza de servicios, por lo que $\mathrm{X}$ y $\mathrm{M}$ incluyen las exportaciones e importaciones de bienes y servicios. 
cia del sector público implica aumentar $\mathrm{G}$ a la ecuación (2), pero además parte del ingreso recibido por el sector privado es transferido al Gobierno vía los impuestos (T). Esto lleva al concepto de ingreso disponible (Yd), de modo que:

$Y d=Y-T$

Como el ingreso disponible se destina al consumo o al ahorro, entonces:

$Y d=C+S$

Combinando (3) y (4) se obtiene lo siguiente:

$C+S=Y d=Y-T$

De aquí puede despejarse $\mathrm{C}=\mathrm{Y}-\mathrm{T}-\mathrm{S}$ y reemplazarse en la ecuación (1):

$P I B=Y=Y-T-S+I+G+X-M$

Si se reagrupa la ecuación (6), se obtiene una identidad fundamental, que representa un punto de partida importante para comprender las crisis externas.

$S-I=(G-T)+(X-M)$

Para entender por qué un país puede tener un déficit en cuenta corriente o brecha externa, la identidad (1) puede reordenarse de la siguiente manera:

$C C=(X-M)=P I B-(C+I+G)$

La cuenta corriente de un país (CC) es la diferencia entre lo que el país produce (PIB) y lo que gasta $(C+I+G)$, lo que, como se ha visto, se denomina absorción. En consecuencia, si PIB > $A$, entonces $C C>0$, con lo cual el país tendría un superávit en la cuenta corriente. De manera análoga, si $\mathrm{PIB}<\mathrm{A}$, entonces, el país produce menos de lo que compra, por lo que la diferencia tiene que importarse ( $y$, por ende, financiarse con recursos del exterior); entonces, $\mathrm{CC}<0$ y el país tendrá un déficit en cuenta corriente.
Si al lector le produce cierta confusión las relaciones anteriores, no debe olvidar que el producto es igual al ingreso, por lo que lo único que se está sosteniendo es que si el país gasta más de lo que le ingresa, la diferencia no solo implica que aumentan las importaciones, sino que también deben financiarse. De lo contrario, ¿cómo podría gastar más que los ingresos en un determinado período?

Otra manera de entender la cuenta corriente es comprender que es igual a la diferencia entre el ahorro interno y la inversión interna. Para el caso de un individuo, como se ha visto, el ahorro es igual a la diferencia entre el ingreso y el consumo. En el caso de un país, el consumo es realizado tanto por el sector privado, C, como por el sector público, G. Por lo tanto:

$S=P I B-C-G$

Sustituyendo (9) en (8), se obtiene:

$\mathrm{CC}=\mathrm{S}-\mathrm{I}$

Para simplificar la explicación de la identidad (10), supongamos inicialmente que I = 0 y que $G$ $=0$. Entonces, si $\mathrm{C}>\mathrm{PBI}$ (que es igual al ingreso), $\mathrm{S}<0$. La cuenta corriente $(\mathrm{CC})$ del individuo sería igual a su ahorro $(\mathrm{CC}=\mathrm{S}<0)$. Por lo tanto, un individuo con un déficit en su cuenta corriente tendrá un nivel negativo de ahorro.

Si se extiende el análisis a un país y se mantiene el supuesto de que $\mathrm{I}=0$, el país con déficit en cuenta corriente estaría consumiendo $(C+G)$ más de lo que está produciendo. De la identidad (9), si $(C+G)>P I B$, entonces, $S=C C<0$.

Ahora veamos el efecto que se produce al levantar el supuesto con respecto de la inversión. Si se asume que $\mathrm{I}>0$ y que además $\mathrm{I}>\mathrm{S}$, entonces, de la identidad (10) se deduce que $\mathrm{CC}<0$. Si tomamos primero el caso del individuo, su ingreso sería menor que su gasto, por lo que tendría un déficit en cuenta corriente 0 , lo que es lo mismo, ahorraría menos de lo que invierte. 
La misma idea es válida para un país. Si I > S, entonces, el PIB es menor que el gasto para consumo e inversión $(C+I+G)$. De este modo, el exceso de gasto (absorción) sobre el ingreso 0 , de manera equivalente, el exceso de inversión sobre el ahorro implica que el país tiene un déficit en cuenta corriente.

Ahora bien, ¿es negativo tener brecha externa? La realidad nos muestra un hecho contundente: los países que atravesaron por una crisis externa (como México en 1994, varios países asiáticos en 1997 y Estados Unidos en 2007) tenían altos niveles de déficit en cuenta corriente. En otras palabras, siguiendo las identidades explicadas, gastaban más de lo que les ingresaba o salían de sus economías más dólares de los que entraban. ¿Qué implicancias tiene ello?; ¿hasta qué punto es sostenible?

Una implicancia es que el país tiene que financiar la diferencia o, lo que es lo mismo, endeudarse. Si el país tiene una situación inicial caracterizada por una riqueza neta positiva (definida como la diferencia entre los activos externos y las deudas con el exterior), el déficit llevará a una reducción de la misma (en el sentido que usará su riqueza previa para financiar el exceso de gasto). Si la tendencia a mantener déficit en cuenta corriente persiste en el tiempo, llegará un momento en que la riqueza neta se agotará y el país (sea por medio del sector público o el sector privado) tendrá que endeudarse, convirtiéndose en deudor neto. De este modo, el aumento en la deuda externa será igual al déficit en la cuenta corriente de cada año.

En consecuencia, los países con un alto stock de deuda externa deben haber tenido antes, altos déficit en cuenta corriente, que originaron la acumulación de esa deuda. Lo anterior ha sido observable en casos ya lejanos, como en México en 1994 y Asia Oriental en 1997; así como en casos recientes, como en los Estados Unidos en 2007. A partir de cierto momento, la acumulación cada vez mayor de deuda externa, sea pública o priva$\mathrm{da}$, se torna insostenible.
Las afirmaciones anteriores pueden ser analizadas a partir de las identidades macroeconómicas, lo que permite diferenciar el ahorro privado (Sp) del ahorro público (Sg), constituyendo la suma de ambos el ahorro nacional o ahorro interno (S). De este modo:

$\mathrm{S}=\mathrm{Sp}+\mathrm{Sg}$

A su vez, $\mathrm{Sp}=\mathrm{PIB}-\mathrm{T}-\mathrm{C}=$ Ahorro privado, donde $T$ son los impuestos recaudados netos de transferencias (TR) y el pago de intereses por la deuda pública (iD); TX son los impuestos brutos. Entonces:

$T=T X-T R-i D$

$\mathrm{T}$ mide todos los ingresos y gastos del gobierno (pues las transferencias y el pago de intereses pueden ser a favor de los contribuyentes) no incluidos en $\mathrm{G}$ (que son las compras de bienes y servicios por parte del gobierno). De este modo, el ahorro público (Sg) se define como la diferencia entre los ingresos $(T)$ y gastos del gobierno $(G)$.

$G-T=G-T X+T R+i D=-S g$

o lo que es lo mismo:

$\mathrm{Sg}=\mathrm{T}-\mathrm{G}$

Como el ahorro privado ( $\mathrm{Sp}$ ) es igual a: $\mathrm{Sp}=\mathrm{PIB}$ - $T$ - $C$, entonces, puede reemplazarse PIB por la identidad fundamental (1), de modo que:

$S p=C+I+G+C C-T-C$, de donde:

$S p=I+G-T+C C$

La identidad (15) combina el ahorro privado (Sp) con el déficit fiscal y la cuenta corriente. En ella se observa que el ahorro privado es una fuente de nuevo capital, en el sentido de que puede usarse en tres fines: 
- Compra de valores de empresas, que financia nuevas inversiones en plantas y equipos, es decir, aumentan I.

- Compra de activos financieros externos, que financia un superávit en la cuenta corriente (CC), si CC > 0. Esto lleva a una acumulación de activos internacionales netos. $\mathrm{Si} \mathrm{CC}<0$, el ahorro privado no es suficiente para financiar I ni el déficit fiscal. En consecuencia, se requiere de ahorro externo (endeudamiento externo) para financiar el exceso de demanda de fondos de las empresas (para inversión) o el Gobierno (para financiar el déficit fiscal).

- Compra de valores gubernamentales, que financian el déficit fiscal, lo que se denomina deuda interna.

De esta manera, el Gobierno y el sector privado pueden competir en el mercado de capitales para captar los ahorros privados. Si el Gobierno capta más ahorro privado, entonces, el sector privado necesariamente capta menos, lo que significa que queda menos ahorro interno para financiar inversión privada. Por lo tanto, un déficit fiscal (G-T) debe tener como contrapartida alguna combinación de mayor ahorro, menor inversión o un déficit en cuenta corriente (véase la identidad 7).

¿Es negativo que un país tenga un déficit en cuenta corriente? La pregunta es válida, pues los países que han enfrentado crisis externas tenían como elemento común un creciente déficit en cuenta corriente. Sin embargo, la respuesta es que el déficit puede ser bueno o malo, sostenible o no, asunto que depende de los factores que originen el déficit.

Aunque parezca un razonamiento circular, el déficit puede ser sostenible en la medida que no exista una crisis externa. Esta última puede aparecer en la forma de una crisis cambiaria o una crisis de deuda externa.

Una crisis cambiaria es una rápida depreciación de la moneda (aumento brusco del tipo de cambio nominal) y/o una rápida pérdida de reservas del Banco Central, y puede ocurrir por las expectativas de los agentes económicos. Si los individuos creen que el tipo de cambio, por alguna razón, subirá en el futuro, entonces, se apresuran a comprar dólares y precipitan el incremento del tipo de cambio. La razón que origina este comportamiento puede ser válida o no, pero el hecho concreto es que mediante la compra de dólares y el consiguiente aumento en el tipo de cambio, los mismos agentes económicos validan sus expectativas.

Una crisis de deuda ocurre cuando un país es incapaz de obtener financiamiento externo para repagarla o también cuando un país declara una moratoria unilateral (como México en 1982 o Rusia en 1998). El país (sea el sector público o el privado) no puede pagar sus obligaciones con el exterior y puede optar por no pagar (escenario que lo aislaría del mercado internacional de capitales) o renegociar y comprometerse a pagar bajo otras condiciones. En este último caso, el país tiene que ajustarse, reduciendo su gasto interno (absorción) para generar un superávit en la cuenta corriente y así obtener los recursos necesarios. Puesto en términos más simples y haciendo la analogía con un individuo, si este ha gastado por encima de sus ingresos, llega un momento en que tiene que gastar menos y/o aumentar sus ingresos para poder pagar sus deudas.

En consecuencia, como una primera aproximación, puede decirse que un déficit en cuenta corriente es sostenible si se puede mantener, sin que ocurra una crisis cambiaria ni una crisis de deuda. Sin embargo, la ausencia de sostenibilidad se observa luego de que los hechos ocurren, es decir, cuando el país ya hizo crisis. De ahí la necesidad de examinar las características de una economía que indican que una crisis puede ocurrir.

Mediante un reordenamiento de la identidad (15), $C C=S p-I-(G-T)$, puede verse que existen tres causas de un déficit en cuenta corriente: un aumento en la inversión interna, una caída en el ahorro privado y/o un aumento del 
déficit fiscal ${ }^{17}$. En la medida que cada caso es distinto, a continuación se analizará el impacto de cada uno de ellos sobre la cuenta corriente, tomando en cuenta que el déficit genera endeudamiento, para poder ser financiado.

\section{a. Un aumento de la inversión interna}

Supongamos que el comportamiento de un país se asemeja al de una empresa, que ha observado oportunidades rentables de inversión, pero que tiene un bajo nivel de ahorro, por lo que decide endeudarse. Para ello, puede acudir al mercado internacional de capitales, al sistema bancario interno o emitir acciones que son adquiridas por los agentes económicos. El endeudamiento será la política adecuada, en la medida que sirva para financiar proyectos rentables; es decir, que el retorno de la inversión sea mayor que el costo del endeudamiento. Una parte de los ingresos futuros generados por el proyecto servirá para pagar la deuda contraída.

El mismo argumento es válido para un país. Imaginemos un país rico en recursos naturales que ha implementado un conjunto de reformas estructurales, luego de haber estabilizado la economía. Entonces, puede producirse un boom de inversión, al aumentar las expectativas de un crecimiento económico futuro en combinación con una alta rentabilidad de nuevos proyectos de inversión. Si el país en cuestión tiene un bajo nivel de ahorro, resulta adecuado recurrir al ahorro externo para financiar el exceso de inversión interna sobre el ahorro interno. El ingreso de capitales, atraído por las expectativas favorables respecto del futuro de la economía, habría actuado como fuente de financiamiento externo, lo que implicará un aumento de la deuda con el exterior.

El país (o específicamente las empresas, que son las que toman las decisiones) puede endeudarse, mediante la atracción de capitales, por alguna de las siguientes formas:
- Tomar préstamos de los bancos extranjeros.

- Prestarse de los bancos internos, quienes a su vez se endeudan con bancos externos. En este caso puede aparecer el riesgo cambiario, si los bancos se endeudan con el exterior en dólares y prestan en el interior en moneda nacional.

- Mediante la emisión de nuevas acciones, que son compradas por inversionistas externos.

- Por la emisión de bonos, comprados por inversionistas externos.

- La nueva inversión puede hacerse de manera directa por empresas extranjeras, es decir, inversión extranjera directa (IED). Este caso no constituye estrictamente deuda externa, pues el país no adquiere ningún compromiso de pagos con el exterior, pero sí constituye ingreso de capitales que financia un mayor gasto de la economía.

En todos estos casos, el déficit en cuenta corriente $(\mathrm{CC}=\mathrm{S}-\mathrm{I}<0)$ está financiado por alguna forma de ahorro externo. En la medida que financia proyectos rentables de inversión, genera la capacidad futura de repago de los préstamos externos.

Sin embargo, ese argumento general amerita varias observaciones. En primer lugar, es positivo, si las nuevas inversiones se realizan en el sector transable, es decir, aquel sector que produce bienes que se pueden exportar en el futuro. De este modo, el déficit actual se convierte en un superávit futuro. Si la inversión se orienta al sector no transable (por ejemplo, el sector construcción), se estarían produciendo bienes que en el futuro no se pueden exportar, por lo que la capacidad de repago futura podría enfrentar dificultades. Un ejemplo de este último caso está relacionado con el boom en los precios de los bienes raíces, previo a la crisis, que se produjo tanto en Asia Oriental en 1997 como en los Estados Unidos en el primer quinquenio del siglo XXI.

17. Esta conclusión también puede extraerse de la identidad (7). 
En segundo lugar, toda empresa sabe que es óptimo endeudarse, si el retorno de la inversión es mayor que el costo del endeudamiento. Si una firma se endeuda en exceso e invierte en proyectos de dudosa rentabilidad, tarde o temprano tendrá pérdidas, lo que generará una crisis financiera, si el argumento se extiende para todo el país. Por lo tanto, la segunda observación está relacionada con la calidad de la inversión.

¿Cómo puede explicarse un boom de inversión en proyectos de baja calidad? Los factores pueden ser diversos, pero, por lo general, depende de la estructura de incentivos existente. En el caso de la crisis de 2007, jugaron dos factores: en primer lugar, el cambio en el modelo de la industria bancaria; de originar un crédito para «mantenerlo» en sus balances a originar un crédito para «revenderlo» a otras instituciones financieras, a partir de entidades que permitían registrarlo fuera del balance. En otras palabras, si un banco otorga un préstamo, digamos un crédito hipotecario, y luego lo vende a un banco de inversión, entonces, transfiere el riesgo, con lo cual no tendrá los incentivos para asumir los cuidados necesarios en el momento de otorgar («originar») el préstamo. En segundo lugar, la existencia del riesgo moral (del inglés moral hazard), que significa que las instituciones financieras relativamente grandes piensan que «son muy grandes para quebrar», por lo que en caso de problemas, el Gobierno evitaría su caída. Esta especie de "garantía implícita» lleva a comportamientos irresponsables.

\section{b. Una caída en el ahorro interno}

El déficit en cuenta corriente puede originarse en una caída en el ahorro privado (México 19901994 y los Estados Unidos en los diez años precios a la crisis) o un aumento en el déficit fiscal (América Latina en la década de 1980). Una reducción del ahorro interno debido a una caída del ahorro público (déficit fiscal) es, en términos potenciales, más peligrosa que una disminución del ahorro privado. La razón estriba en que los ahorros privados aumentan cuando se incrementan los ingresos futuros, mientras que los déficit fiscales son más complejos de revertir. En los años 1970, en América Latina, los déficit fiscales llevaron no solo a un crecimiento del déficit en cuenta corriente, sino también a un aumento del stock de deuda externa. Hacia 1982, el incremento del cociente deuda externa/PIB originó que los gobiernos no pudieran cumplir con sus obligaciones, explotando la crisis de la deuda, que obligó a efectuar renegociaciones atadas a costosos programas de ajuste.

En síntesis, el déficit en cuenta corriente debe ser motivo de preocupación cuando el boom de consumo es el resultado de una rápida liberalización del mercado doméstico de capitales, que aumenta el acceso al crédito a agentes económicos que antes tenían restricciones para endeudarse.

\section{c. Otros factores}

Además de los elementos descritos, la sostenibilidad del déficit en cuenta corriente también depende de otros factores macroeconómicos. En primer lugar, altas tasas de crecimiento económico suponen altas tasas de inversión y, por ende, de ahorro, lo que hace menos dependiente al país. Sin embargo, este patrón no encaja con las experiencias de Chile (1979-1981), México (19771981) y Asia Oriental (1997). En los tres casos, las tasas de crecimiento que existían antes de la crisis eran mayores que $7 \%$. El problema de estos casos radicó en el optimismo excesivo originado por las mismas tasas (en el sentido de que se mantendrían en el largo plazo), que llevó a un boom del consumo y de la inversión, (demanda interna) con la consiguiente reducción del ahorro interno, algo similar a la crisis de los Estados Unidos. El aumento exagerado del consumo y de la inversión generó un aumento de la deuda externa, que en determinado momento se tornó insostenible, en especial porque se trataba de endeudamiento privado de corto plazo. Un segundo factor está en la naturaleza del déficit comercial (diferencia entre exportaciones e importaciones de bienes), pues puede reflejar problemas estructurales de competitividad de la economía. En ese caso, el déficit en cuenta corriente es menos sostenible. 
En tercer lugar, el ratio X/PIB es un indicador de la capacidad futura de servir la deuda externa. Por último, el tamaño del déficit en cuenta corriente, porque los episodios de crisis han coincidido con brechas externas mayores que $4 \%$ del PIB. Este indicador se convierte en una señal de alarma, si está financiado con capitales volátiles.

El uso de la información económica: una «lectura» del entorno

En el cuadro 1 se presenta la evolución del PIB por el lado de la oferta en variaciones porcentuales, respecto del mismo período del año previo. La utilidad del cuadro radica, al menos, en dos razones: en primer lugar, observar la tendencia global de evolución del PIB en comparación, en todo momento, con igual período del año previo. En segundo lugar, permite determinar qué sectores son la locomotora de la economía; dicho de otro modo, si la cifra global es un promedio del comportamiento de los distintos sectores, los sectores que «jalan el promedio hacia arriba» son los responsables de la cifra de variación del PIB global.

Una lectura del cuadro 1 nos lleva a las siguientes conclusiones:

- La economía peruana creció a tasas altas, con relación a la tendencia histórica, en los años 2007 y 2008 (véase la última fila).
- El proceso de crecimiento es interrumpido a partir del primer trimestre de 2009. Ello es una consecuencia de los impactos de la crisis financiera internacional (véase la última fila).

- En el ámbito sectorial, comparando el primer trimestre de 2009 con el primer trimestre de 2008, se observan reducciones en casi todos los sectores de la economía.

- La desaceleración continuó en el segundo trimestre de 2009, con lo cual la evolución del PIB (comparada con igual trimestre del año 2008) fue negativa $(-1,1 \%)$. El dato no es menor, pues el resultado negativo ocurrió después de 28 trimestres consecutivos de aumentos en el PIB.

¿Cómo explicar los resultados anteriores? A partir de la evolución del PIB por tipo de gasto, información que se presenta en el cuadro 2, responde a la siguiente pregunta: ¿quién compra lo producido? En principio existen dos opciones: vender en el mercado interno (demanda interna) o en el externo (demanda externa o exportaciones). A su vez, la demanda interna está dividida en cuatro componentes: consumo público, consumo privado, inversión pública e inversión privada. De esta manera, el PIB aumenta o disminuye en función de los cambios ocurridos en alguno(s) de los cinco componentes mencionados.

\section{Cuadro 1}

\section{Perú: PIB por sectores productivos}

(Variaciones porcentuales respecto del mismo período del año anterior)

\begin{tabular}{|l|r|r|r|r|r|}
\hline & 2007 & 2008 & 1 T-09 & 2 T-09 & 1Sem-09 \\
\hline Agropecuario & 3,3 & 7,2 & 4,2 & 1,3 & 2,5 \\
Pesca & 6,9 & 6,2 & $-19,8$ & $-6,8$ & $-9,5$ \\
Minería e hidrocarburos & 2,7 & 7,6 & 3,7 & 0,6 & 2,1 \\
Manufactura & 10,8 & 8,7 & $-5,3$ & $-11,1$ & $-8,3$ \\
Electricidad y agua & 8,5 & 7,7 & 1,2 & 0,2 & 0,7 \\
Construcción & 16,6 & 16,5 & 5,1 & $-1,1$ & 2,0 \\
Comercio & 9,7 & 13,0 & 0,4 & $-2,1$ & $-1,0$ \\
Otros servicios & 9,6 & 8,9 & 4,7 & 3,6 & 4,2 \\
\hline PIB global & 8,9 & 9,8 & 1,8 & $-1,1$ & 0,3 \\
\hline
\end{tabular}

Fuente: Banco Central de Reserva del Perú, <http://www.bcrp.gob.pe>. 


\section{Cuadro 2}

\section{Perú: PIB por tipo de gasto}

(Variaciones porcentuales respecto del mismo período del año anterior)

\begin{tabular}{|l|r|r|r|r|}
\hline & 2007 & 2008 & 1 T-09 & 2T-09 \\
\hline Demanda interna & 11,8 & 12,3 & $-0,8$ & $-5,5$ \\
a. Consumo privado & 8,3 & 8,8 & 3,7 & 1,9 \\
b. Consumo público & 4,5 & 4,0 & 8,2 & 8,9 \\
c. Inversión privada & 23,4 & 25,6 & 1,8 & $-20,8$ \\
d. Inversión pública & 18,2 & 41,9 & 22,2 & 12,9 \\
\hline Demanda externa (exportaciones) & 6,2 & 8,2 & $-3,0$ & $-3,1$ \\
\hline Importaciones & 21,3 & 19,9 & $-13,7$ & $-24,0$ \\
\hline PIB & 8,9 & 9,8 & 1,8 & $-1,1$ \\
\hline
\end{tabular}

Fuente: Banco Central de Reserva del Perú, <http://www.bcrp.gob.pe>.

El cuadro 2 aclara el panorama. Pueden extraerse las siguientes conclusiones:

- Tanto la demanda interna como la demanda externa disminuyen en los dos primeros trimestres del año 2009, algo que no había ocurrido en los dos años previos. Por lo tanto, lo que explicó el comportamiento del PIB en los años 2007 y 2008 fue la evolución de ambas demandas.

- En los años 2007 y 2008, en la evolución de la demanda interna crecieron todos sus componentes; mientras que la desaceleración se observa a partir del primer trimestre de 2009, en particular en la inversión privada y en el comercio internacional, pues tanto las exportaciones como las importaciones disminuyen. Recordemos que en un modelo de desarrollo basado en el mercado, la inversión privada y el comercio externo son los motores de la economía.

- Las caídas en la inversión privada han sido reemplazadas con aumentos de la inversión pública, dentro del Ilamado "Plan de Estímulo Económico», implementado en 2008 para enfrentar los efectos negativos de la crisis financiera internacional. Por esa razón, solo el consumo y la inversión pública aumentan en el segundo trimestre de 2009.

Sin embargo, ¿cómo explicar la caída en ambas demandas a partir del primer trimestre de 2009, que marca un punto de inflexión en el avance económico? Ello nos lleva al siguiente paso: el análisis de la economía internacional, dado que son los impactos de la crisis financiera los que explican los resultados internos. A manera de referencia y con fines metodológicos, en el cuadro 3 se presenta lo ocurrido con la economía mundial y las proyecciones para el año 2009. Ciertamente, para una empresa que exporta a determinado país, el siguiente paso sería analizar el entorno del país en cuestión de manera similar al «estudio de cifras» realizado para el Perú en los cuadros 1 y 2 .

Las 33 economías avanzadas son responsables del $55,3 \%$ del producto mundial y del $65,3 \%$ de las exportaciones mundiales; mientras que las 139 economías emergentes y en desarrollo explican la diferencia. Estados Unidos es la economía más importante del mundo, pues produce el $20,7 \%$ del PIB mundial, la Eurozona produce el $15,7 \%$ del total y Japón, el 6,4\%. Las tres zonas en conjunto mostraron una desaceleración en 2008 (en comparación con 2007) y una caída en el estimado para 2009. Por el lado de las economías emergentes, China explica el $11,1 \%$ del PIB global, mientras que América Latina solo el 8,6\% (Fondo Monetario Internacional 2009). La caída en el comercio mundial, con una contracción de $-12,2 \%$ en 2009 , es histórica. 


\section{Cuadro 3}

Proyecciones del panorama económico mundial

(Variaciones porcentuales)

\begin{tabular}{|l|c|c|c|}
\hline & 2007 & 2008 & $2009^{1 /}$ \\
\hline Producto mundial & 5,1 & 3,1 & $-1,4$ \\
1. Economías avanzadas (33 países) & 2,7 & 0,8 & $-3,8$ \\
Estados Unidos & 2,0 & 1,1 & $-2,6$ \\
Eurozona & 2,7 & 0,8 & $-4,8$ \\
Japón & 2,3 & $-0,7$ & $-6,0$ \\
2. Economías emergentes (139 países) & 8,3 & 6,0 & 1,5 \\
Europa Oriental (11 países) & 5,4 & 3,0 & $-5,0$ \\
Rusia & 8,1 & 5,6 & $-6,5$ \\
China & 13,0 & 9,0 & 7,5 \\
India & 9,4 & 7,3 & 5,4 \\
América Latina & 9,4 & 7,3 & 5,4 \\
Volumen de comercio mundial & 7,2 & 2,9 & $-12,2$ \\
\hline
\end{tabular}

1/: Cifras estimadas.

Fuente: Fondo Monetario Internacional (2009), Tabla 1.1

El deterioro de la economía mundial explica los resultados de la economía peruana de manera casi simétrica. El párrafo anterior muestra que al caer las economías cuyo aporte al PIB mundial es mayor, los impactos negativos en el resto de las economías actúan como un choque exógeno contra el cual es poco lo que se puede hacer. Ahora, volvamos al cuadro 2. La menor demanda interna se explica por la retracción de los inversionistas ante los resultados de la economía mundial (cuadro 3). La reducción en la demanda externa se deriva de la contracción en el comercio mundial.

De esta manera, el entorno económico interno es explicado por la evolución de la economía mundial. El ejemplo, con datos de la realidad, sirve para construir el análisis del entorno económico desde dentro y hacia fuera.

Hacia una síntesis del análisis del entorno: los pasos por seguir

Con el objetivo de proporcionar al lector una secuencia ordenada del estudio del entorno, a continuación se presenta una propuesta de los pasos por seguir. Desde luego, solo se incluye variables de entorno económico, pues no es posible incluir a todas las variables, razón por la cual se trata de una aproximación que facilita el análisis del mismo.

- Definir el período relevante; por lo general, se recomienda tomar en cuenta los tres años previos. Las proyecciones pueden realizarse de dos modos: asumiendo que se mantiene la tendencia o alterándola, dado algún cambio representativo, como podría significar una reorientación de la visión económica por un cambio de gobierno.

- Analizar la evolución del PIB por el lado de la oferta, con el objetivo de observar las tendencias sectoriales.

- Observar la evolución de la inflación, para ver si existe estabilidad monetaria o no. En el caso peruano, la meta inflacionaria desde el año 2007 es $2 \%$ anual $+/-1 \%$.

- Relacionar la evolución de la brecha externa con el comportamiento del PIB, con el fin de observar si las variaciones en el PIB son explicadas por mayores importaciones $y / 0$ un crecimiento de las exportaciones.

- Si en el paso anterior se verifica que la brecha externa aumenta con el PIB, entonces, la ra- 
zón está en mayores importaciones. Ello requiere de un mayor ingreso de capitales. Caso contrario, debe observarse que el incremento en el PIB conduce a una reducción de la brecha externa. En el ámbito internacional, la brecha externa se considera sostenible si no excede el $4 \%$ del PIB anual.

- Analizar la evolución del PIB por el lado del gasto, para observar si la demanda interna y/o la externa son los motores. En ambos casos, el comportamiento de las mismas está condicionado por la evolución de la economía internacional. En el primero, por medio de la evolución de la inversión privada; y en el segundo, directamente, por las exportaciones.

- Los movimientos en la demanda (interna o externa) conducen al análisis de la economía internacional y su evolución. Como ha sido demostrado, en 2009, la reducción en las demandas para el caso peruano tuvo su origen en la evolución de la economía internacional.

\section{Conclusiones}

La empresa opera dentro de un entorno económico que es complejo, dinámico, cambiante y poco predecible. Comprenderlo es básico para mejorar la toma de decisiones. Sin embargo, ello se enfrenta por lo menos a dos obstáculos: en primer lugar, el «lenguaje» usado por los especialistas parece ser complejo; en segundo lugar, la rapidez con la que fluye la información respecto de los cambios en el entorno, no solo nacional sino internacional, altera los pronósticos empresariales y genera que proyectos que eran rentables ya no lo sean y viceversa. De ahí que el manejo de los conceptos básicos utilizados por los analistas sea una necesidad dentro de la empresa. No es casual que los de planes de negocios incorporen un análisis del entorno (no solo económico).

La presente investigación ha revisado algunos de los indicadores económicos más usados, cuya recurrencia obliga a tener un conocimiento de los mismos. La evolución del PIB, como un indicador del nivel de la actividad económica, puede ser analizada desde varias ópticas. Aquí, se han revisado dos de ellas: por el lado de la oferta (sectores productivos) y por el lado de la demanda (o tipo de gasto). La primera permite analizar qué sectores constituyen la locomotora de una economía; mientras que la segunda explica por qué lo son. En este punto aparece la conexión con el entorno económico internacional, que explica la evolución de variables relevantes, como la inversión privada, las exportaciones y las importaciones. Otras variables del entorno económico son: la inflación y la brecha externa. Dada la evolución económica mundial de las últimas dos décadas, el énfasis ha estado en las crisis externas. La relación entre ellas se ha formalizado con la explicación detallada de las identidades macroeconómicas, cuyo análisis aparece en la primera aplicación empírica.

Las predicciones de lo que "ocurrirá con la economía» enfrentan limitaciones, pues el ser humano, cuyo comportamiento resulta casi impredecible, está ubicado en el centro del análisis. No obstante, la economía busca encontrar patrones y tendencias; por ejemplo, si un país (individuo) enfrenta una brecha externa (gasta más de lo que le ingresa) y la financia aumentando el nivel de deuda externa (cubre la diferencia con endeudamiento), llegará un momento en que, de mantener el comportamiento descrito, no podrá pagar y hará crisis.

Finalmente, los directivos de las empresas y los economistas operan en un contexto de incertidumbre. La crisis financiera internacional ha mostrado justamente esas limitaciones, pero ha mostrado un patrón recurrente en la historia. Aun así, vuelve a ocurrir. La incertidumbre del entorno no cambiará, pero ello no impide que el hecho de conocerlo, cada vez más, aumentará la capacidad de mejorar la toma de decisiones empresariales. 


\section{Referencias}

BOYER, R. y D. DRACHE

1996 States against Markets. The Limits of Globalization, Londres: Routledge.

\section{DEARDOFF, A. y R. STEIN}

2000 What the Public Should Know about Globalization and the World Trade Organization? Research Seminar in International Economics Discussion Paper $\mathrm{N}^{\circ}$ 460. Michigan: The University of Michigan.

FONDO MONETARIO INTERNACIONAL

2009 «Tabla 1.1». En: World Economic Outlook Update. Fecha de consulta: 09/07/2009. $<$ http://www.imf.org $>$.

PARODI TRECE, C.

2009 Globalización y crisis financieras internacionales. Cuarta reimpresión. Lima, Perú: Universidad del Pacífico, Centro de Investigación.

2008 Globalización, ¿de qué y para qué? Lecciones de la historia. Primera reimpresión. Lima, Perú: Universidad del Pacífico, Centro de Investigación.
PROGRAMA DE LAS NACIONES UNIDAS PARA EL DESARROLLO-PNUD

2009 Informe sobre el desarrollo humano 20072008. Madrid: Grupo Mundi-Prensa. Disponible en <http://www.undp.org>.

SCHULDT, J.

1998 «Desmitificando el concepto de globalización». En: Instituto Latinoamericano de Investigaciones Sociales (eds.). Globalización: mito y realidad. Quito, Ecuador: Tramasocial editores, pp. 9-38.

\section{Páginas Webs}

BANCO CENTRAL DE RESERVA DEL PERÚ

$<$ http://www.bcrp.gob.pe>.

NATIONAL BUREAU OF ECONOMIC RESEARCH $<$ http://www.nber.org>. 